\title{
Incidence and Oncological Implications of Previously Undetected Tumor Multicentricity Following Pancreaticoduodenectomy for Pancreatic Adenocarcinoma in Patients Undergoing Salvage Pancreatectomy
}

\author{
ANDREAS ANDREOU ${ }^{1,2}$, FRITZ KLEIN ${ }^{1}$, ROSA B. SCHMUCK ${ }^{1}$, DANIELA LEE ${ }^{1}$, \\ MARIANNE SINN ${ }^{3}$, TIMM DENECKE ${ }^{4}$, JOHANN PRATSCHKE ${ }^{1}$ and MARCUS BAHRA ${ }^{1}$ \\ Departments of ${ }^{1}$ Surgery, ${ }^{3}$ Hematology, Oncology and Tumor Immunology, and ${ }^{4}$ Radiology, and ${ }^{2}$ Berlin School \\ of Integrative Oncology, Charité Medical University of Berlin, Virchow Campus Hospital, Berlin, Germany
}

\begin{abstract}
Background: The risk for multicentricity of pancreatic adenocarcinoma remains unclear and the question whether pancreaticoduodenectomy represents sufficient oncological treatment for patients with ductal adenocarcinoma of the head of the pancreas needs further investigation. Patients and Methods: Clinicopathological data of patients who underwent pancreaticoduodenectomy for pancreatic adenocarcinoma between 2005 and 2015 were assessed and the incidence of tumor multicentricity among patients who required salvage pancreatectomy within 90 postoperative days was evaluated. Results: Pancreaticoduodenectomy was performed in 1,005 patients. Sixty-two patients (6\%) suffered a major postoperative complication (pancreatic fistula/anastomotic leak/bleeding) after resection of the head of the pancreas, requiring salvage pancreatectomy. Histological examination of the pancreatic remnant in patients with ductal adenocarcinoma $(n=19)$ revealed multicentric carcinoma in two patients, resulting in an incidence of $11 \%$ for tumor multicentricity. Preoperative cross-sectional imaging failed to identify tumor multicentricity in these patients. Additionally, two patients with pancreatic intraepithelial neoplasia and two with neuroendocrine tumor were identified. Conclusion: The
\end{abstract}

Presented at the 46th Annual Meeting of the American Pancreatic Association, San Diego, November 2015.

Correspondence to: Andreas Andreou, MD, Department of Surgery, Campus Charité Mitte and Campus Virchow-Klinikum, Charité Universitätsmedizin Berlin, Augustenburger Platz 1, 13353 Berlin, Germany. Tel: +49 30450652274, Fax: +49 30450552900, e-mail: andreas.andreou@charite.de

Key Words: Pancreatic cancer, tumor multicentricity, salvage pancreatectomy. incidence of previously undetected multicentric adenocarcinoma among patients undergoing salvage pancreatectomy in our study was surprisingly high. This finding suggests that the role of total pancreatectomy for pancreatic head cancer, as well as the current strategies for postoperative tumor surveillance, should be re-evaluated in order to provide the best oncological approach and prolonged survival for patients with ductal adenocarcinoma.

Pancreaticoduodenectomy (PD) is currently considered the preferable therapeutic option with curative intent for patients with adenocarcinoma of the pancreatic head (1). Recent advances in the perioperative management of these patients may be responsible for the reduction of postoperative mortality rates to under $5 \%$ and improvement of 5-year overall survival rates to $20 \%$ following PD for pancreatic adenocarcinoma $(2,3)$. Advanced preoperative imaging enables accurate detection of the tumor burden and detailed planning of the surgical procedure (4). Surgical techniques have evolved, guaranteeing minimum blood loss and low rates of anastomotic-related complications $(5,6)$. Multidisciplinary management of patients with pancreatic adenocarcinoma has been established in major cancer centers, allowing more patients to receive multimodal treatment combining systemic therapy with surgery $(3,7)$. The use of adjuvant chemotherapy with gemcitabine, as well as with modern regimens such as FOLFIRINOX (8) and gemcitabine/nabpaclitaxacel (9), has contributed to further improvements in survival (3).

Despite these significant advances in the treatment of pancreatic adenocarcinoma, tumor recurrence following radical resection remains high, thereby limiting long-term survival (10). Several factors have been identified as being associated with diminished disease-free survival including tumor stage, lymph node metastasis (11), and tumor involvement of the 
resection margins (12). However, the impact of multicentricity of pancreatic adenocarcinoma on recurrence following PD remains controversial. Whereas earlier studies reported an incidence of tumor multicentricity beyond the resection line of a usual Kausch-Whipple procedure to be as high as $40 \%$ (13, 14), immunohistochemical and genomic analyses of pancreatectomy specimens indicated that the frequency of multicentric tumor localization among patients with pancreatic cancer is significantly lower $(6-8 \%)(15,16)$.

Therefore, the aim of this study was to investigate the incidence of tumor multicentricity in a cohort of patients who required salvage pancreatectomy following PD for ductal adenocarcinoma of the pancreas. We additionally aimed to identify factors associated with an increased risk for multifocal tumor localization in the pancreas.

\section{Patients and Methods}

Patient inclusion criteria. Following Institutional Review Board approval (EA2/035/14), we collected and reviewed the clinicopathological data of all consecutive patients who underwent PD between 2005 and 2015 in our Department. Patients who underwent salvage procedure with resection of the pancreatic remnant due to major complication including pancreatic fistula, anastomotic leak, and bleeding within 90 days following PD for ductal adenocarcinoma of the pancreatic head were included in the analysis. Patients with inoperable disease and those who underwent palliative procedure were excluded from the database.

Preoperative assessment. Preoperative patient evaluation included medical history, physical examination, serum laboratory tests, imaging studies, and anesthesia evaluation. For the diagnosis and staging of pancreatic cancer, an endoscopic retrograde cholangiopancreatography with endosonography and brush biopsy were regularly performed. Cross-sectional imaging was used to define the location and extent of the tumor with respect to major vascular involvement (celiac trunk/superior mesenteric artery or greater than $180^{\circ}$ involvement/thrombosis of the superior mesenteric vein/portal venous axis) (1), to identify lymph node metastases and to exclude distant metastatic disease. Fluorodeoxyglucose-positronemission tomography and diagnostic laparoscopy were additional tools used to clarify resectability in selected cases. Tumor resectability was discussed and decided by a multidisciplinary tumor board, which consisted of pancreatic surgeons, gastroenterologists, medical oncologists, and specialized radiologists.

Surgical procedure. Resection of the pancreatic head was performed using a standardized pylorus-preserving PD or Kausch-Whipple procedure as described before by our group (17). Reconstruction of the pancreatic remnant included either an anastomosis to the dorsal gastric wall as a pancreatogastrostomy or to a dissected jejunal loop as an end-to-side-pancreatojejunostomy (17). The remaining bile duct was reconstructed performing an end-to-side hepaticojejunostomy and gastrointestinal passage was restored by Roux-en-Y gastrojejunostomy (17). Perianastomotic drains were regularly placed to monitor for pancreatic fistula, anastomotic leaks and postoperative hemorrhage, and nasogastric tubes were placed routinely in all patients.
Postoperative management. After PD, patients were admitted to an Intensive Care Unit specialized for patients undergoing hepatopancreatobiliary surgery and were closely monitored for relevant postoperative complications including anastomotic leak, pancreatic fistula, bleeding, intra-abdominal infection, and organ failure. Nasogastric decompression and nil per os diet with concomitant parenteral nutrition was established until anastomotic leak was excluded using a routine radiocontrast agent swallow examination on the fifth postoperative day. Unremarkable anastomotic evaluation was followed by removal of the nasogastric tube and oral diet was gradually advanced. Pancreatic fistula and anastomotic leak were identified based on the observation of remarkable discharge through the perianastomotic drain and routine examination of the lipase levels in the drained fluids. Postoperative pancreatic fistulas (POPF) were classified according to the criteria of the International Study Group on Pancreatic Fistula and patients were treated according the severity grade (A, B, or C) with antibiotic therapy, therapeutic somatostatin analogs, total parenteral nutrition, transfusion, prolonged maintenance of drains, additional percutaneous drainage, continuous saline irrigation of the abdomen, and in more severe cases of septic deterioration of the patient or acute hemorrhage with revision surgery including salvage completion pancreatectomy (18). In the case of an unremarkable postoperative course, the perianastomotic drains were removed on the fifth postoperative day if the discharge was less than $500 \mathrm{cc}$ per day.

Postoperative chemotherapy was administered to all patients with ductal adenocarcinoma regardless of the status of the resection margins following presentation at the multidisciplinary institutional tumor board. Adjuvant treatment was initiated within 6 weeks following PD, provided that wound healing was complete and the patient's clinical condition was accordingly suitable for systemic therapy.

Histological evaluation. All resected specimens were histologically examined to define the entity of the pancreatic neoplasm and to describe the tumor stage according to the TNM classification. Standardized protocols were used for the histological examination of PD specimens from patients with pancreatic adenocarcinoma in order to evaluate the pancreatic transection margin, the bile duct and stomach/duodenal margins, as well as the circumferential soft-tissue margins, for the presence of tumor cells. R0 resection was defined as a complete tumor resection with surgical margins $>1 \mathrm{~mm}$ microscopically negative for tumor cells (19). Furthermore, the pancreatic remnant following salvage pancreatectomy was histologically examined for the presence of multicentric adenocarcinoma or other neoplastic lesions.

Statistical analysis. Quantitative and qualitative variables are expressed as the median (range) and frequency, respectively. Chisquared or Fisher's exact test and the Mann-Whitney $U$-test were used to compare categorical and continuous variables, as appropriate, and identify factors associated with tumor multicentricity in univariate analysis. The following factors were therefore analyzed: sex, age at resection, type of surgical procedure, time to salvage pancreatectomy, American Society of Anesthesiologists (ASA) physical status, duration of resection, need for intraoperative transfusions, tumor size in the pancreatic head, T stage, lymph node involvement, tumor grading, Union for International Cancer Control (UICC) stage, lymphangiosis carcinomatosa, venous invasion, 
perineural invasion, resection margins, remnant pancreatitis, pancreatic fistula, anastomotic leak, postoperative bleeding, postoperative chemotherapy, type of pancreatic anastomosis, duration of hospital stay, and 90-day postoperative mortality.

$p$-Values of 0.05 or less were considered statistically significant. Statistical analyses were performed using the SPSS software package, version 22 (IBM, Armonk, NY, USA).

\section{Results}

Patient characteristics. During the study period, 1,005 patients underwent PD for pancreatic cancer, ampullary cancer, distal bile duct cancer, duodenal cancer, chronic pancreatitis, neuroendocrine tumors, and other indications in $45 \%, 8 \%, 14 \%, 2 \%, 12 \%, 4 \%$, and $15 \%$ of cases, respectively. Sixty-two patients $(6 \%)$ suffered a grade $\mathrm{C}$ POPF requiring salvage revision surgery with completion pancreatectomy following PD.

Among patients with ductal adenocarcinoma of the pancreatic head $(n=452), 19$ underwent salvage resection of the pancreatic remnant $(4 \%)$ (Figure 1). The clinicopathological data of these patients are summarized in Tables I and II. The majority of patients were male $(n=12)$ and the median age at resection was 64 years (43-83). Seventeen patients underwent pylorus-preserving PD and two patients a Kausch-Whipple procedure. A leak of the pancreatic anastomosis occurred in 18 patients (nine patients after pancreatogastrostomy $v s$. nine patients after pancreatojejunostomy, $p=0.330$ ). Acute hemorrhage due to arrosive bleeding that led to remnant pancreatectomy involved 12 patients. The median time to salvage pancreatectomy was 8 (range $=3-24)$ days.

Histological examination of the pancreatic remnant in patients with ductal adenocarcinoma $(n=19)$ revealed multicentric carcinoma in two patients resulting in an incidence of $11 \%$ for tumor multicentricity. The size of the pancreatic tumor in the remnant was 4 and $5 \mathrm{~mm}$, respectively and the distance between the resection margin and the multicentric tumor in the tail of the pancreas was 3 and $4 \mathrm{~cm}$, respectively. Preoperative cross-sectional imaging failed to identify tumor multicentricity in these patients. Additionally, two patients with pancreatic intraepithelial neoplasia and two with neuroendocrine tumor were identified.

Factors associated with tumor multicentricity. Factors associated with the presence of multicentric pancreatic cancer are presented in Table $\mathrm{I}$ and included $\mathrm{T}$ stage $(p=0.014)$, the presence of lymph node metastases $(p=0.05)$, UICC stage $(p=0.17)$, and lymphagiosis carcinomatosa $(p=0.012)$. In conclusion, patients with advanced $\mathrm{T}$ stage and lymph node involvement resulting in an advanced UICC stage, and patients with lymphagiosis carcinomatosa were found to be at higher risk of presenting with multicentric

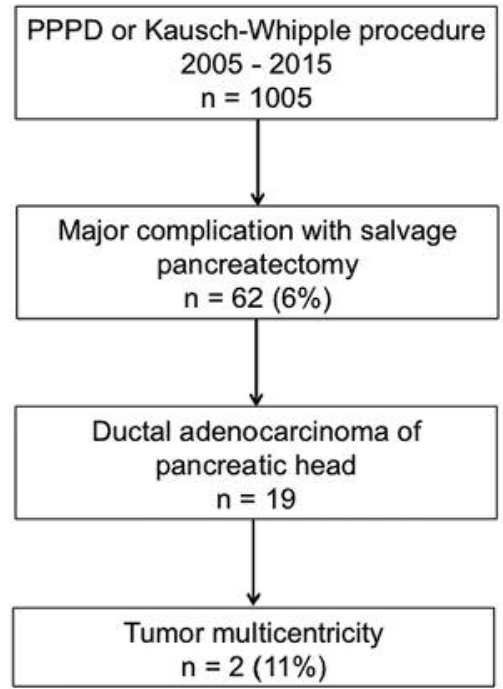

Figure 1. Study cohort including 19 patients who underwent salvage pancreatectomy for postoperative pancreatic fistula $C$ following pancreaticoduodenectomy for adenocarcinoma of the head of the pancreas. PPPD: Pylorus-preserving pancreaticoduodenectomy.

ductal adenocarcinoma of the pancreas. Both patients with multicentric tumor were diagnosed with perineural tumor invasion and had poorly or moderately differentiated cancer, however, these factors did not reach statistical significance.

\section{Discussion}

Previous studies have reported on the presence of tumor multicentricity in patents with pancreatic adenocarcinoma, providing controversial rates ranging between $6 \%(15,16)$ and $40 \%(13,14)$. Therefore, the question of whether PD represents a sufficient oncological treatment for patients with ductal adenocarcinoma of the head of the pancreas requires further investigation, and the role of total pancreatectomy in the treatment of pancreatic head cancer needs to be revisited.

Our study examined the incidence of tumor multicentricity among 19 patients who underwent salvage pancreatectomy after PD for pancreatic cancer and identified two with previously undetected cancer focus beyond the PD resection line, leading to a multicentricity rate of $11 \%$. Surprisingly, two $(11 \%)$ additional patients had a pancreatic intraepithelial neoplasia and two (11\%) further patients had a neuroendocrine tumor in the tail of the pancreas. These findings underline the concern that a significant number of patients with resectable pancreatic cancer may be exposed to a higher risk for tumor recurrence following PD with curative intent due to incomplete resection of undetected multifocal sites of cancer (20). The presence of abnormal findings in the pancreatic remnant in six patients overall 
Table I. Clinicopathological characteristics related to initial pancreaticoduodenectomy for pancreatic cancer in 19 patients undergoing subsequent salvage pancreatectomy due to postoperative pancreatic fistula grade C.

\begin{tabular}{|c|c|c|c|c|}
\hline Characteristics & $\begin{array}{l}\text { All patients } \\
\quad \mathrm{N}=19\end{array}$ & $\begin{array}{l}\text { No tumor multicentricity } \\
\qquad \mathrm{N}=17\end{array}$ & $\begin{array}{l}\text { Tumor multicentricity } \\
\qquad \mathrm{N}=2\end{array}$ & $p$-Value* \\
\hline Gender, n (\%) & & & & 0.683 \\
\hline Male & $12(63)$ & $11(65)$ & $1(50)$ & \\
\hline Female & $7(37)$ & $6(35)$ & $1(50)$ & \\
\hline Median age at resection (range), years & $64(43-83)$ & $64(43-83)$ & $61(57-67)$ & 0.634 \\
\hline Surgical procedure, n (\%) & & & & 0.608 \\
\hline PPPD & $17(90)$ & $15(88)$ & $0(0)$ & \\
\hline Kausch-Whipple procedure & $2(10)$ & $2(12)$ & $2(100)$ & \\
\hline ASA physical status, $\mathrm{n}(\%)$ & & & & 0.522 \\
\hline I & $1(6)$ & $1(6)$ & $0(0)$ & \\
\hline II & $10(50)$ & $8(46)$ & $2(100)$ & \\
\hline III & $5(28)$ & $5(29)$ & $0(0)$ & \\
\hline IV & $3(16)$ & $3(19)$ & $0(0)$ & \\
\hline Median duration of resection (range), min & $317(241-678)$ & $336(257-678)$ & $248(241-256)$ & 0.032 \\
\hline Need for intraoperative transfusion, $\mathrm{n}(\%)$ & $12(67)$ & $12(73)$ & $0(0)$ & 0.140 \\
\hline Pancreatic anastomosis, $\mathrm{n}(\%)$ & & & & 0.115 \\
\hline Pancreatogastrostomy & $9(47)$ & $7(41)$ & 100 & \\
\hline Pancreatojejunostomy & $10(53)$ & $10(59)$ & $0(0)$ & \\
\hline Median tumor size (range), $\mathrm{mm}$ & $30(10-55)$ & $26(10-55)$ & $33(30-35)$ & 0.422 \\
\hline T Stage, n $(\%)$ & & & & 0.014 \\
\hline $\mathrm{T} 1$ & $1(6)$ & $1(6)$ & $0(0)$ & \\
\hline $\mathrm{T} 2$ & $0(0)$ & $0(0)$ & $0(0)$ & \\
\hline $\mathrm{T} 3$ & $17(89)$ & $16(94)$ & $1(50)$ & \\
\hline $\mathrm{T} 4$ & $1(5)$ & $0(0)$ & $1(50)$ & \\
\hline Lymph node metastases, n (\%) & $7(37)$ & $5(29)$ & $2(100)$ & 0.050 \\
\hline Tumor grading, n (\%) & & & & 0.836 \\
\hline G1 & $1(6)$ & $1(6)$ & $0(0)$ & \\
\hline $\mathrm{G} 2$ & $12(61)$ & $10(59)$ & $1(50)$ & \\
\hline G3 & $6(33)$ & $6(35)$ & $1(50)$ & \\
\hline UICC stage, n (\%) & & & & 0.017 \\
\hline Ia & $2(10)$ & $2(12)$ & & \\
\hline $\mathrm{Ib}$ & $0(0)$ & $0(0)$ & $0(0)$ & \\
\hline IIa & $10(53)$ & $10(59)$ & $0(0)$ & \\
\hline $\mathrm{IIb}$ & $6(32)$ & $5(29)$ & $1(50)$ & \\
\hline III & $1(5)$ & $0(0)$ & $1(50)$ & \\
\hline Lymphangiosis carcinomatosa, $\mathrm{n}(\%)$ & $5(26)$ & $3(19)$ & $2(100)$ & 0.012 \\
\hline Venous invasion, $\mathrm{n}(\%)$ & $1(5)$ & $1(6)$ & $0(0)$ & 0.725 \\
\hline Perineural invasion, $\mathrm{n}(\%)$ & $11(58)$ & $9(53)$ & $2(100)$ & 0.202 \\
\hline Positive resection margins (R1), n (\%) & $4(22)$ & $4(25)$ & $0(0)$ & 0.423 \\
\hline
\end{tabular}

UICC, Union for International Cancer Control; ASA, American Society of Anesthesiologists; PPPD, pylorus-preserving pancreaticoduodenectomy. *Comparison of patients with and without tumor multicentricity.

Table II. Clinicopathological characteristics related to salvage pancreatectomy due to postoperative pancreatic fistula grade $C$ following pancreaticoduodenectomy for pancreatic cancer in 19 patients.

\begin{tabular}{|c|c|c|c|c|}
\hline Characteristics & $\begin{array}{l}\text { All patients } \\
\quad \mathrm{N}=19\end{array}$ & $\begin{array}{l}\text { No tumor multicentricity } \\
\qquad \mathrm{N}=17\end{array}$ & $\begin{array}{l}\text { Tumor multicentricity } \\
\qquad \mathrm{N}=2\end{array}$ & $p$-Value* \\
\hline Pancreatic fistula, n (\%) & $19(100)$ & $17(100)$ & $2(100)$ & 0.990 \\
\hline Leak of pancreatic anastomosis, $\mathrm{n}(\%)$ & $18(95)$ & $16(94)$ & $2(100)$ & 0.725 \\
\hline Postoperative bleeding, $\mathrm{n}(\%)$ & $12(63)$ & $11(65)$ & $1(50)$ & 0.683 \\
\hline Median time to salvage pancreatectomy (range), days & $8(3-24)$ & $10(3-24)$ & $4(3-4)$ & 0.038 \\
\hline Remnant pancreatitis, n (\%) & $19(100)$ & $17(100)$ & $2(100)$ & 0.990 \\
\hline Postoperative chemotherapy, $\mathrm{n}(\%)$ & $8(42)$ & $8(47)$ & $2(100)$ & 0.343 \\
\hline Median duration of hospital stay (range), days & $36(11-208)$ & $36(11-208)$ & $65(26-105)$ & 0.574 \\
\hline 90-Day mortality, n (\%) & $8(42)$ & $8(47)$ & $0(0)$ & 0.182 \\
\hline
\end{tabular}

*Comparison of patients with and without tumor multicentricity. 
$(32 \%)$ indicates that alternative treatment strategies including extended pancreatic resection should be discussed.

Total pancreatectomy was proposed as a treatment strategy to improve outcomes in patients with ductal adenocarcinoma of the pancreas with regard to a considerable risk of tumor multicentricity $(21,22)$. However, subsequent studies showed that total pancreatectomy was associated with higher rates of postoperative morbidity (52\%) and mortality (27\%) (23), and diminished long-term survival (5-year overall survival rate of 9\%) (24) compared to subtotal pancreatectomy (25). Additionally, the diabetic state induced by total pancreatectomy and characterized by complete insulin deficiency, pancreatic polypeptide deficiency, and an absence of functional glucagon $(26,27)$ was associated with reduced quality of life (28), contributing to the consensus during the 1990s that this procedure cannot be established as the standard of care for patients with cancer of the pancreatic head $(20,29)$.

Recent improvements in the perioperative management and surgical technique in total pancreatectomy have increased the safety and feasibility of this procedure and provided acceptable postoperative morbidity (37.6\%) and mortality $(3.7 \%)$ rates (30) among patients undergoing pancreatic surgery. Increased utilization of adjuvant chemotherapy with modern agents (3) has enabled long-term survival (5-year overall survival up to $26 \%$ ) comparable to that of patients undergoing PD (31). Finally, increasing implementation of long-acting insulin formulations and oral pancreatic enzyme supplements has improved the management of endocrine and exocrine pancreas insufficiency, reducing post-pancreatectomy metabolic impairment and allowing for a better quality of life (31). Consequently, total pancreatectomy could regain a position among the surgical tools for the treatment of pancreatic cancer in selected cases, especially in the light of the risk of tumor multicentricity. Further surgical indications including familial pancreatic adenocarcinoma, diffuse intraductal papillary mucinous neoplasms, multiple pancreatic neuroendocrine tumors, and a soft and fragile pancreatic remnant with a high risk for anastomotic leaks may justify an upgrade of the role of total pancreatectomy in pancreatic surgery $(31,32)$. Additionally, the indication of islet autotransplantation has been recently extended for selected patients with malignant disease such as pancreatic or periampullary cancer to improve glycemic control and improve quality of life after total pancreatectomy. However, the risk of disease dissemination associated with this procedure based on the high incidence of multiple undetected tumors in the pancreatic tail should be taken into serious consideration and further investigated in larger studies (33).

Our analysis identified an association between the presence of tumor multicentricity and advanced tumor stage (UICC), including an extended $\mathrm{T}$ stage and the presence of lymph node metastases. Lymphangiosis carcinomatosa was also correlated with an increased risk of multicentric pancreatic cancer. Perineural tumor invasion and reduced tumor differentiation were identified in both patients with multicentric tumor, even though statistical significance was not reached. These findings indicate that tumor multicentricity may represent a marker for unfavorable tumor biology resulting in extended tumor spread. The association between tumor multicentricity and multiple Kirsten rat sarcoma viral oncogene (KRAS) mutations described by Motojima et al. (16) may provide partial explanation for our observation that multicentric lesions were diagnosed in patients with advanced pancreatic cancer, since somatic gene mutations, including those of $K R A S$, have already been associated with aggressive tumor biology, reduced response to systemic therapy, and poor survival following resection $(34,35)$.

Our study is limited by its retrospective nature. Furthermore, we chose to include patients who underwent salvage pancreatectomy following PD for ductal adenocarcinoma, instead of examining the specimens of patients undergoing total pancreatectomy, because this was, in our opinion, a more effective way to identify a random cohort of patients who were selected for PD after preoperative exclusion of continuous tumor growth beyond the PD resection line in order to investigate the frequency of undetected multifocal tumor spread. An additional limitation may be the small number of patients requiring salvage pancreatectomy resulting in only two patients with multicentric cancer. Nevertheless, the multicentricity rate of $11 \%$ was surprisingly high and worthy of discussion of the clinical relevance of this condition. The small number of patients additionally limits the weight of the analysis for factors associated with a higher risk for tumor multicentricity. However, the observation that several surrogate factors for unfavorable tumor biology, such as UICC stage, T stage, $\mathrm{N}$ stage, lymphangiosis carcinomatosa, perineural tumor invasion, and tumor grading correlated with tumor multicentricity allows conclusions to be drawn regarding the need for an optimized perioperative management of patients with extended tumor burden.

In conclusion, this study indicated that the incidence of tumor multicentricity among patients with ductal adenocarcinoma of the pancreas was relatively high, reaching $11 \%$ of the investigated patients, and can therefore not be considered as negligible. Modified treatment strategies such as increased utilization of total pancreatectomy in selected patients and intensified postoperative tumor surveillance in patients undergoing PD for advanced cancer may significantly contribute to the reduction of tumor recurrence and improvement of long-term survival in patients with pancreatic cancer .

\section{Conflicts of Interest}

The Authors declare no conflicts of interest. 


\section{References}

1 Bahra M, Pratschke J, Klein F, Neuhaus P, Boas-Knoop S, Puhl G, Denecke T, Pullankavumkal JR, Sinn M, Riess H and Pelzer $\mathrm{U}$ : Cytoreductive surgery for pancreatic cancer improves overall outcome of gemcitabine-based chemotherapy. Pancreas 44: 930936, 2015

2 Cameron JL, Riall TS, Coleman J and Belcher KA: One thousand consecutive pancreaticoduodenectomies. Ann Surg 244: 10-15, 2006.

3 Oettle H, Neuhaus P, Hochhaus A, Hartmann JT, Gellert K, Ridwelski K, Niedergethmann M, Zulke C, Fahlke J, Arning MB, Sinn M, Hinke A and Riess H: Adjuvant chemotherapy with gemcitabine and long-term outcomes among patients with resected pancreatic cancer: the CONKO-001 randomized trial. JAMA 310: 1473-1481, 2013.

4 Denecke T, Grieser C, Neuhaus P and Bahra M: Radiologic resectability assessment in pancreatic cancer. Rofo 186: 23-29, 2014.

5 Serrano PE, Cleary SP, Dhani N, Kim PT, Greig PD, Leung K, Moulton CA, Gallinger S and Wei AC: Improved long-term outcomes after resection of pancreatic adenocarcinoma: a comparison between two time periods. Ann Surg Oncol 22: 1160-1167, 2015.

6 Keck T, Wellner UF, Bahra M, Klein F, Sick O, Niedergethmann M, Wilhelm TJ, Farkas SA, Borner T, Bruns C, Kleespies A, Kleeff J, Mihaljevic AL, Uhl W, Chromik A, Fendrich V, Heeger K, Padberg W, Hecker A, Neumann UP, Junge K, Kalff JC, Glowka TR, Werner J, Knebel P, Piso P, Mayr M, Izbicki J, Vashist Y, Bronsert P, Bruckner T, Limprecht R, Diener MK, Rossion I, Wegener I and Hopt UT: Pancreatogastrostomy Versus Pancreatojejunostomy for RECOnstruction After PANCreatoduodenectomy (RECOPANC, DRKS 00000767): Perioperative and long-term results of a multicenter randomized controlled trial. Ann Surg 263: 440-449, 2016.

7 Neoptolemos JP, Dunn JA, Stocken DD, Almond J, Link K, Beger H, Bassi C, Falconi M, Pederzoli P, Dervenis C, Fernandez-Cruz L, Lacaine F, Pap A, Spooner D, Kerr DJ, Friess H, Buchler MW and European Study Group for Pancreatic C: Adjuvant chemoradiotherapy and chemotherapy in resectable pancreatic cancer: a randomised controlled trial. Lancet 358 : 1576-1585, 2001.

8 Conroy T, Desseigne F, Ychou M, Bouche O, Guimbaud R, Becouarn Y, Adenis A, Raoul JL, Gourgou-Bourgade S, de la Fouchardiere C, Bennouna J, Bachet JB, Khemissa-Akouz F, Pere-Verge D, Delbaldo C, Assenat E, Chauffert B, Michel P, Montoto-Grillot C, Ducreux M, Groupe Tumeurs Digestives of $\mathrm{U}$ and Intergroup P: FOLFIRINOX versus gemcitabine for metastatic pancreatic cancer. N Engl J Med 364: 1817-1825, 2011.

9 Von Hoff DD, Ervin T, Arena FP, Chiorean EG, Infante J, Moore M, Seay T, Tjulandin SA, Ma WW, Saleh MN, Harris M, Reni M, Dowden S, Laheru D, Bahary N, Ramanathan RK, Tabernero J, Hidalgo M, Goldstein D, Van Cutsem E, Wei X, Iglesias J and Renschler MF: Increased survival in pancreatic cancer with nabpaclitaxel plus gemcitabine. N Engl J Med 369: 1691-1703, 2013.

10 Raut CP, Tseng JF, Sun CC, Wang H, Wolff RA, Crane CH, Hwang R, Vauthey JN, Abdalla EK, Lee JE, Pisters PW and Evans DB: Impact of resection status on pattern of failure and survival after pancreaticoduodenectomy for pancreatic adenocarcinoma. Ann Surg 246: 52-60, 2007.

11 Sierzega M, Popiela T, Kulig J and Nowak K: The ratio of metastatic/resected lymph nodes is an independent prognostic factor in patients with node-positive pancreatic head cancer. Pancreas 33: 240-245, 2006.

12 Rau BM, Moritz K, Schuschan S, Alsfasser G, Prall F and Klar E: R1 resection in pancreatic cancer has significant impact on long-term outcome in standardized pathology modified for routine use. Surgery 152: S103-111, 2012.

13 Ihse I, Lilja P, Arnesjo B and Bengmark S: Total pancreatectomy for cancer. An appraisal of 65 cases. Ann Surg 186: 675-680, 1977.

14 Tryka AF and Brooks JR: Histopathology in the evaluation of total pancreatectomy for ductal carcinoma. Ann Surg 190: 373$381,1979$.

15 Kloppel G, Lohse T, Bosslet K and Ruckert K: Ductal adenocarcinoma of the head of the pancreas: incidence of tumor involvement beyond the Whipple resection line. Histological and immunocytochemical analysis of 37 total pancreatectomy specimens. Pancreas 2: 170-175, 1987.

16 Motojima K, Urano T, Nagata Y, Shiku H, Tsurifune T and Kanematsu T: Detection of point mutations in the Kirsten-ras oncogene provides evidence for the multicentricity of pancreatic carcinoma. Ann Surg 217: 138-143, 1993.

17 Eckardt AJ, Klein F, Adler A, Veltzke-Schlieker W, Warnick P, Bahra M, Wiedenmann B, Neuhaus P, Neumann K and Glanemann M: Management and outcomes of haemorrhage after pancreatogastrostomy versus pancreatojejunostomy. Br J Surg 98: 1599-1607, 2011.

18 Bassi C, Dervenis C, Butturini G, Fingerhut A, Yeo C, Izbicki J, Neoptolemos J, Sarr M, Traverso W, Buchler M and International Study Group on Pancreatic Fistula D: Postoperative pancreatic fistula: an international study group (ISGPF) definition. Surgery 138: 8-13, 2005.

19 Esposito I, Kleeff J, Bergmann F, Reiser C, Herpel E, Friess H, Schirmacher $\mathrm{P}$ and Buchler MW: Most pancreatic cancer resections are R1 resections. Ann Surg Oncol 15: 1651-1660, 2008.

20 Reddy SK, Tyler DS, Pappas TN and Clary BM: Extended resection for pancreatic adenocarcinoma. Oncologist 12: 654663, 2007.

21 Collins JJ Jr., Craighead JE and Brooks JR: Rationale for total pancreatectomy for carcinoma of the pancreatic head. N Engl J Med 274: 599-602, 1966.

22 Lygidakis NJ, Singh G, Bardaxoglou E, Dedemadi G, Sgourakis G, Nestoridis J, Malliotakis A, Pedonomou M, Solomou EK, Safioleas M, Alamani M, Grigorakos L and Merikas EM: Monobloc total spleno-pancreaticoduodenectomy for pancreatic head carcinoma with portal-mesenteric venous invasion. A prospective randomized study. Hepatogastroenterology 51: 427-433, 2004.

23 Ihse I, Anderson $\mathrm{H}$ and Andren S: Total pancreatectomy for cancer of the pancreas: is it appropriate? World J Surg 20: 288293; discussion 294, 1996.

24 Karpoff HM, Klimstra DS, Brennan MF and Conlon KC: Results of total pancreatectomy for adenocarcinoma of the pancreas. Arch Surg 136: 44-47; discussion 48, 2001.

25 Sarr MG, Behrns KE and van Heerden JA: Total pancreatectomy. An objective analysis of its use in pancreatic cancer. Hepatogastroenterology 40: 418-421, 1993. 
26 Doi K, Prentki M, Yip C, Muller WA, Jeanrenaud B and Vranic M: Identical biological effects of pancreatic glucagon and a purified moiety of canine gastric immunoreactive glucagon. J Clin Invest 63: 525-531, 1979.

27 Muller WA, Brennan MF, Tan MH and Aoki TT: Studies of glucagon secretion in pancreatectomized patients. Diabetes 23 : 512-516, 1974.

28 Dresler CM, Fortner JG, McDermott $\mathrm{K}$ and Bajorunas DR: Metabolic consequences of (regional) total pancreatectomy. Ann Surg 214: 131-140, 1991.

29 Reddy S, Wolfgang CL, Cameron JL, Eckhauser F, Choti MA, Schulick RD, Edil BH and Pawlik TM: Total pancreatectomy for pancreatic adenocarcinoma: evaluation of morbidity and longterm survival. Ann Surg 250: 282-287, 2009.

30 Johnston WC, Hoen HM, Cassera MA, Newell PH, Hammill CW, Hansen PD and Wolf RF: Total pancreatectomy for pancreatic ductal adenocarcinoma: review of the National Cancer Data Base. HPB 18: 21-28, 2016.

31 Hartwig W, Gluth A, Hinz U, Bergmann F, Spronk PE, Hackert $\mathrm{T}$, Werner $\mathrm{J}$ and Buchler MW: Total pancreatectomy for primary pancreatic neoplasms: renaissance of an unpopular operation. Ann Surg 261: 537-546, 2015.
32 Heidt DG, Burant C and Simeone DM: Total pancreatectomy: indications, operative technique, and postoperative sequelae. $\mathrm{J}$ Gastrointest Surg 11: 209-216, 2007.

33 Balzano G, Maffi P, Nano R, Zerbi A, Venturini M, Melzi R, Mercalli A, Magistretti P, Scavini M, Castoldi R, Carvello M, Braga M, Del Maschio A, Secchi A, Staudacher C and Piemonti L: Extending indications for islet autotransplantation in pancreatic surgery. Ann Surg 258: 210-218, 2013.

34 Shin SH, Kim SC, Hong SM, Kim YH, Song KB, Park KM and Lee YJ: Genetic alterations of K-RAS, p53, c-ERBB-2, and DPC4 in pancreatic ductal adenocarcinoma and their correlation with patient survival. Pancreas 42: 216-222, 2013.

35 Eser S, Schnieke A, Schneider G and Saur D: Oncogenic KRAS signalling in pancreatic cancer. Br J Cancer 111: 817-822, 2014.

Received July 9, 2017

Revised August 2, 2017

Accepted August 4, 2017 